\title{
Mathematical and numerical analysis for a model of growing metastatic tu- mors
}

\author{
Dominique Barbolosi * _ Assia Benabdallah ** _ Florence \\ Hubert $^{* *}$ - Federico Verga ${ }^{*}, * *$
}

* Université de la Méditerranée - UPRES EA 3286 - IFR 125 Physiopathologie Humaine de Marseille 27, boulevard Jean Moulin, 13385 Marseille Cedex 5, France.

** Université de Provence, LATP (UMR CNRS 6632),

39 rue F. Joliot Curie, 13453 Marseille Cedex 13, France

\{assia,fhubert,verga\}@latp.univ-mrs.fr, dominique.barbolosi@neuf.fr

Abstract. In cancer diseases, the appearance of metastases is a very pejorative forecast. Chemotherapies are systemic treatments which aim at the elimination of the micrometastases produced by a primitive tumour. The efficiency of chemotherapies closely depends on the protocols of administration. Mathematical modeling is an invaluable tool to help in evaluating the best treatment strategy. Iwata and al [11] proposed a partial differential equation (PDE) that describes the metastatic evolution of an untreated tumour. In this article, we conducted a thorough mathematical analysis of this model. Particularly, we provide an explicit formula for the growth rate parameter, as well as a numerical resolution of this PDE. By increasing our understanding of the existing model, this work is crucial for further extension and refinement of the model. It settles down the framework necessary for the consideration of drugs administration effects on tumour developpment.

Keywords: Von Foerster equation, semigroup approach, asymptotic behaviour, characteristic scheme, metastatic tumors

\section{Introduction}

Primary tumors after reaching a critical volume may be at the origin of several metastatic tumors disseminated in the human body. At early stages, metastatic tumors are of small size and not detectable by medicinal apparatus, like in the case of the breast cancer, but evidence for the existence of occult micrometastases at diagnosis is overwhelming [10]. Metastases grow rapidly, escape any therapeutic treatment and often lead to the patient's death. This is the fate of an uncontrolled disease process. 
Because metastases, the systemic therapy is the only therapeutic treatment which may have an impact on the problem of disseminated tumors [9]. The adequate systemic therapy is chemotherapy based on the combination of cytotoxic, cytostatic or other biologic agents. Any chemotherapeutic treatment must be initiated before intensive disseminating process, in order to prevent the emergence of micrometastases. It is the so-called adjuvant chemotherapy given despite undetectable tumor masses. This is the by the chemotherapy disturbance of tumor growth process.

Disease monitoring joint to chemotherapy constitutes not only a high complex but also constrained process because of the undesirable toxic adverse events that limit the applicability of chemotherapy. Optimal management of cancer requires today thorough and upgraded tools integrating current information about cancer cell kinetic pharmacokinetics and efficacy-toxicity pharmacodynamics.

Since several years numerous authors attempted to optimize anticancer chemotherapy by using mathematical modeling [3]. Dose-dense schedules may have an advantage over conventional schedules of drug administration [13], and we have already developed mathematical models computing the features of an administration protocol so that the size of primary tumor is minimized while the toxicity effects are controlled. These features concern the drug amounts (intensification) and their distribution over a treatment cycle (densification). This approach was applied to the metastatic breast cancer in phase I trial [7].

Recently, disease models were also developed describing the dissemination dynamics of metastatic tumors [11]. In this line, Iwata et al. developed a metastatic disease model as a partial differential equation with initial and boundary conditions. Starting from a primary tumor with one cell, this equation calculates the colony size distribution $v(x, t)$ of metastatic tumors with cell numbers $x$ at time $t$. The authors obtain an analytical solution of this equation using Laplace transform; however the obtained solution is not numerically tractable.

In contrast to all these developments, no models are available for the disease and treatment joint processes. Especially for micrometrastases, these models could describe the number and sizes of tumors below the detection threshold, predict the behavior of metastases in patients and thus allow the design of adjuvant chemotherapy.

Accordingly, we propose to revisit the Iwata's model in order to incorporate the effect of chemotherapy and pharmacokinetics and pharmacodynamics of antitumor agents. The purpose of this paper will be a thorough mathematical analysis of the so obtained new partial differential equation. This analysis will be followed by the presentation of a numerical scheme of resolution. Aiming to prevent the emergence of the micrometastases, accordingly the Iwata's model has to be modified to incorporate the effect of chemotherapy in order to to de- 
sign an administration protocol which will minimize the number of metastases, by using the method of optimization developed in [7].

Reader's guide

We precise in section 2 the model under study: a Von Foerster equation with Gompertzian growth. It is a linear transport equation with a non local boundary condition combined with a singular source. The mathematical analysis of such an equation is developped in section 3 . We, in particular, derive the asymptotic behaviour of the solution. We exhibit a Malthus parameter $\lambda_{0}$ that gives the growth rate of metastatic sites. This explicit formula that gives this parameter shows a deep dependance on clinical characteristic data of the patient. We propose in section 4 an algorithm to approximate the solutions of such an equation. We give a theoritical validation of the numerical model for long time intervals. We finally give in section 4 some numerical results. Our results are first compared to the ones of [11]. Numerical simulations also confirm the theoritical asymptotic growth of the number of metastases. We conclude this section by a sensibility analysis with respect to the clinical characteristic data of the patient.

\section{Presentation of the model}

We present in this section the mathematical model describing the dynamics of the metastatic colony size distribution introduced in [11]. In this model we suppose that the primary tumor is generated by a single cell at $t=0$ and grows with the rate $g(x)$ per unit time where $x$ is the tumor size represented by the number of cells in the tumor. The growing tumor emits metastatic cells with the rate $\beta(x)$. Each metastatic cell develops into a new tumor, which also grows at rate $g(x)$ and emits new nuclei of metastasis just as the primary tumor does. Let $v(x, t)$ represent the colony size distribution with cell number $x$ at time $t$, actually $v(x, t) d x$ means the number of metastatic tumors whose sizes range from $x$ and $x+d x$ at time $t$. In the case considered here we suppose that the nuclei of colonization are located far enough from each other so that their ranges do not overlap for a long time period. The dynamics of the colony size distribution is given by the following Mac-Kendrick Von Foerster equation:

$$
\left\{\begin{array}{l}
\frac{\partial}{\partial t} v(x, t)+\frac{\partial}{\partial x}[g(x) v(x, t)]=0, x \in[1, b), t \geq 0 \\
g(1) v(1, t)=\int_{1}^{b} \beta(x) v(x, t) d x+\beta\left(x_{p}(t)\right) \\
v(0, x)=0 .
\end{array}\right.
$$

We have to deal with a transport equation with a non local boundary condition and an initial data equals to zero which supposes that there is no metastatic 
tumor at time $t=0$. The boundary condition means that the number of metastatic cells newly created per unit time at time $t$ is the total rate of occurences of metastases due to metastatic tumors (corresponding to the integral term) and the primary tumor.

In the Iwata's model the data $x_{p}, g$ and $\beta$ are precised as follows.

1) The number of cells $x_{p}(t)$ in the primary tumor at time $t$ is the solution of the Cauchy problem:

$$
\left\{\begin{array}{l}
\frac{d}{d t} x_{p}(t)=g\left(x_{p}(t)\right) \\
x_{p}(0)=1
\end{array}\right.
$$

2) A Gompertzian growth rate $g$ is adopted:

$$
g(x)=a x \ln \left(\frac{b}{x}\right),
$$

where $a$ denotes a growth rate constant and $b>1$ the maximum tumor size.

We can solve explicitely the equation (2) which gives:

$$
x_{p}(t)=b^{1-e^{-a t}} .
$$

3) The colonization rate $\beta(x)$ is choosen as:

$$
\beta(x)=m x^{\alpha},
$$

where $m$ is the colonization coefficient and $\alpha$ is the fractal dimension of blood vessels infiltrating the tumor. The parameter $\alpha$ expresses how the blood vessels geometrically distribute in or on a tumor. If the vascularity is superficial the fractal dimension $\alpha$ is assigned to be $2 / 3$ because we suppose that the tumor has the shape of a sphere hence the surface area is proportional to $2 / 3$. Else if the vascularization is homogeneously distributed in the whole tumor, $\alpha$ is supposed to be equal to 1 .

\section{Mathematical analysis of the Von Foerster equation with Gompertzian growth}

This section is devoted to the mathematical analysis of System 1. We are going to use a semigroup approach. This will give the existence and uniqueness of solutions as well as the long time behaviour of these solutions. Apart from the construction of the semigroup, the main ideas can be found in [15],[12]. We would like also to refer to [6] for another approach. 
Let us consider the equation (1) in a more general case where the initial condition is non zero:

$$
\left\{\begin{array}{l}
\frac{\partial}{\partial t} v(x, t)+\frac{\partial}{\partial x}[g(x) v(x, t)]=0, x \in(1, b), t \geq 0 \\
g(1) v(1, t)=\int_{1}^{b} \beta(x) v(x, t) d x+f(t), t>0 \\
v(x, 0)=v_{0}(x)
\end{array}\right.
$$

\subsection{Existence of solutions}

In the sequel, we suppose that $g$ is given by $(3), \beta$ by $(5)$ and $f(t)=\beta\left(x_{p}(t)\right)$ where $x_{p}$ the number of cells in primary tumor is defined in (4). In particular, we have

$$
\int_{1}^{b} \frac{\beta(x)}{g(x)} d x=+\infty .
$$

Let us introduce the operator $A$ and its domain:

$$
\begin{aligned}
A & =-\partial_{x}(g(x) .), \\
D(A) & =\left\{v \in L^{1}(1, b), g v \in W^{1,1}(1, b), \lim _{x \rightarrow b^{-}}(g v)(x)=0,(g v)(1)=\int_{1}^{b} \beta(x) v(x) d x\right\} .
\end{aligned}
$$

In order to prove the existence of solutions for the considered equation (6), we first need to state with some spectral properties of the operator $A$.

\subsubsection{Spectral properties of $A$}

We derive in this section some properties of the point spectrum $\sigma_{p}(A)$, the set of the eigenvalues of $(A, D(A))$.

Theorem 1. There exists a unique real eigenvalue $\left.\lambda_{0} \in\right] 0,+\infty[$ of the operator $(A, D(A))$. More precisely :

$$
m<\lambda_{0}<m b^{\alpha}
$$

Moreover there exists $\varepsilon>0$ such that

$$
\sigma_{p}(A) \subset\left\{0<\operatorname{Re} \lambda<\lambda_{0}-\varepsilon\right\} \cup\left\{\lambda_{0}\right\}
$$

Proof.

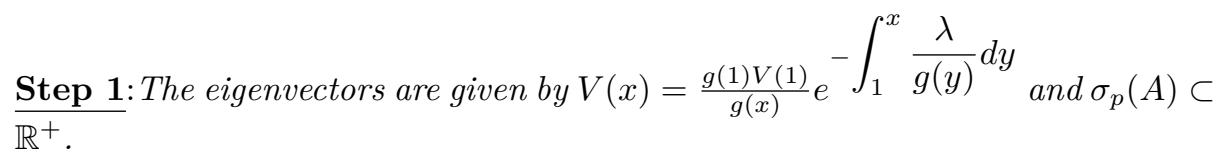


We recall that $\lambda \in \sigma_{p}(A)$ if and only if there exists, $V \neq 0$ such that

$$
\left\{\begin{array}{l}
V \in D(A) \\
A V=\lambda V, \text { in }(1, b)
\end{array}\right.
$$

The solutions of the differential equation

$$
A V=\partial_{x}(g(x) V)=-\lambda V, \text { in }(1, b)
$$

are given by:

$$
V(x)=\frac{g(1) V(1)}{g(x)} e^{-\int_{1}^{x} \frac{\lambda}{g(y)} d y} .
$$

The boundary condition

$$
g(1) V(1)=\int_{1}^{b} \beta(x) V(x) d x
$$

then reads

$$
g(1) V(1)=\int_{1}^{b} g(1) V(1) \frac{\beta(x)}{g(x)} e^{-\int_{1}^{x} \frac{\lambda}{g(y)} d y} d x
$$

First of all, notice that the function $g V$ defined in $(9)$ is in $W^{1,1}(1, b)$ and $\lim _{x \rightarrow b^{-}}(g V)(x)=0$ if and only if $\operatorname{Re} \lambda>0$. Moreover, direct calculations show that

$$
e^{-\lambda \int_{1}^{x} \frac{d y}{a y \ln \frac{b}{y}}}=\left(\frac{\ln \frac{b}{x}}{\ln b}\right)^{\frac{\lambda}{a}}
$$

and therefore

$$
V(x)=\frac{V(1)}{x}\left(\frac{\ln \frac{b}{x}}{\ln b}\right)^{\frac{\lambda}{a}-1} .
$$

Thus $V \in L^{1}(1, b)$ if and only if $\operatorname{Re} \lambda>0$. Therefore, to be in the spectrum of $(A, D(A)), \operatorname{Re} \lambda$ has to be greater than 0 .

We can remark that if $V(1)=0$ then $V=0$, so $V(1) \neq 0$ to ensure that $V \neq 0$ and $\operatorname{dim}(\operatorname{Ker}(A-\lambda I))=1$.

We deduce that $\lambda \in \mathbb{C}$ is an eigenvalue of $(A, D(A))$ if and only if $\operatorname{Re} \lambda>0$ and from (10):

$$
F(\lambda):=\int_{1}^{b} \frac{\beta(x)}{g(x)} e^{-\int_{1}^{x} \frac{\lambda}{g(y)} d y} d x=1 .
$$

Let us introduce

$$
G(x)=\int_{1}^{x} \frac{d y}{g(y)}
$$


Thanks to the properties of $g$ the function $G$ is strictly increasing, $\mathcal{C}^{1}$ and one to one from $(1, b)$ to $(0,+\infty)$. As $G^{\prime}(x) \neq 0, \forall x \in(1, b), G$ is a $\mathcal{C}^{1}$ diffeomorphism. We can rewrite $F(\lambda)$ as follows:

$$
F(\lambda)=\int_{0}^{+\infty} \beta\left(G^{-1}(y)\right) e^{-\lambda y} d y .
$$

Then, setting $\theta=\beta \circ G^{-1}$, we remark that:

$$
F(\lambda)=\int_{0}^{+\infty} \theta(y) e^{-\lambda y} d y
$$

$F$ is the Laplace transform of the function $\theta=\beta \circ G^{-1} \in L^{\infty}(0, \infty)$, we deduce that $F$ is well defined and holomorphic (Laplace transform property) in the complex half plane $\Omega=\{\lambda \in \mathbb{C}, \operatorname{Re} \lambda>0\}$. Thus the solutions of the equation $F(\lambda)=1$ are all isolated. We have:

$$
V\left(G^{-1}(x)\right)=g(1) V(1) G^{\prime}\left(G^{-1}(x)\right) e^{-\lambda x} \text { for all } x \in(0,+\infty) .
$$

\section{Step 2:}

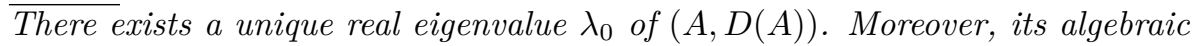
multiplicity is 1 .

We will look at the solutions of (11) on the real line. Remark that $F$ is continuous and strictly decreasing on $\mathbb{R}^{+}$with $F(0)>1$ thanks to (7) and:

$$
\lim _{\lambda \rightarrow+\infty} F(\lambda)=0 .
$$

Therefore by the intermediate value theorem, (11) admits a unique solution $\lambda_{0} \in \mathbb{R}^{+}$.

As $\lambda_{0}>0$, we prove in the last step that $\operatorname{ker}\left(A-\lambda_{0} I\right)=\mathbb{R} V$ for $V$ defined by (9). In the sequel, we fix $V(1)>0$.

Let us now show $\operatorname{ker}\left(A-\lambda_{0} I\right)^{2}=\operatorname{ker}\left(A-\lambda_{0} I\right)=\mathbb{R} V$. A function $\varphi \in$ $\operatorname{ker}\left(A-\lambda_{0} I\right)^{2}$ if and only if $\varphi \in D(A)$ and there exists $c \in \mathbb{R}$ such that:

As previously this system is equivalent to:

$$
\left\{\begin{array}{l}
A \varphi=\lambda_{0} \varphi-c V, \text { in }(1, b) \\
g(1) \varphi(1)=\int_{1}^{b} \beta(x) \varphi(x) d x
\end{array} .\right.
$$

$$
\begin{array}{r}
\partial_{x}(g(x) \varphi)=-\lambda_{0} \varphi+c V, \text { in }(1, b) \\
g(1) \varphi(1)=\int_{1}^{b} \beta(x) \varphi(x) d x
\end{array}
$$


Solving the differential equation (13) we obtain for $\varphi$ :

$$
\varphi(x)=\frac{g(1) \varphi(1)}{g(x)} e^{-\int_{1}^{x} \frac{\lambda_{0}}{g(y)} d y}+\frac{c}{g(x)} \int_{1}^{x} e^{-\int_{\tau}^{x} \frac{\lambda_{0}}{g(y)} d y} V(\tau) d \tau .
$$

One can deduce (since $F\left(\lambda_{0}\right)=1$ ):

$$
\int_{1}^{b} \beta(x) \varphi(x) d x=g(1) \varphi(1)+c \int_{1}^{b} \frac{\beta(x)}{g(x)} \int_{1}^{x} e^{-\int_{\tau}^{x} \frac{\lambda_{0}}{g(y)} d y} V(\tau) d \tau d x
$$

In order to fulfill the condition (14) the condition:

$$
c \int_{1}^{b} \frac{\beta(x)}{g(x)} \int_{1}^{x} e^{-\int_{\tau}^{x} \frac{\lambda_{0}}{g(y)} d y} V(\tau) d \tau d x=0
$$

is required. As $\beta, g, V$ are positive functions on $[1, b)$ we deduce that $c=0$ and:

$$
\varphi(x)=\frac{g(1) \varphi(1)}{g(x)} e^{-\int_{1}^{x} \frac{\lambda_{0}}{g(y)} d y}=\delta V(x),
$$

with $\delta=\frac{\varphi(1)}{V(1)}$, the conclusion follows. Therefore the algebraic multiplicity of $\lambda_{0}$ is 1 .

It remains to prove (8). From (11), one has :

$$
F\left(\lambda_{0}\right)=\int_{0}^{+\infty} \beta\left(G^{-1}(x)\right) e^{-\lambda_{0} x} d x=1 .
$$

As $m<\beta(x)<m b^{\alpha}$ for $x \in(1, b)$, one gets that

$$
\frac{m}{\lambda_{0}}<1<\int_{0}^{+\infty} m b^{\alpha} e^{-\lambda_{0} x} d x=\frac{m b^{\alpha}}{\lambda_{0}}
$$

so

$$
m<\lambda_{0}<m b^{\alpha} .
$$

As we will see in the sequel, $\lambda_{0}$ gives the asymptotic behaviour.

\section{Step 3:}

There is a unique eigenvalue whose real part is equal to $\lambda_{0}$.

Let $\lambda_{0}$ the real solution of $F\left(\lambda_{0}\right)=1$. If $\lambda=\lambda_{0}+i \alpha, \alpha \in \mathbb{R}$, is an eigenvalue of $(A, D(A))$ since $\theta=\beta\left(G^{-1}\right)$ is real valued we have:

$$
F(\lambda)=1 \text { if and only if }\left\{\begin{array}{l}
\int_{0}^{\infty} \theta(x) e^{-\lambda_{0} x} \cos (\alpha x) d x=1 \\
\int_{0}^{\infty} \theta(x) e^{-\lambda_{0} x} \sin (\alpha x) d x=0
\end{array}\right.
$$


Since $F\left(\lambda_{0}\right)=1$, one can deduce that:

$$
\int_{0}^{\infty} \theta(x) e^{-\lambda_{0} x}(1-\cos (\alpha x)) d x=0
$$

Considering that $\theta>0$ almost everywhere, this can be true if and only if $1-\cos (\alpha x)=0$, for all $x \in \mathbb{R}$. Therefore $\alpha=0$.

Step 4:

There $i s$ no eigenvalue whose real part is bigger than $\lambda_{0}$.

Indeed if $\lambda \in\left\{\operatorname{Re} \lambda>\lambda_{0}\right\}$ then $|F(\lambda)|<F\left(\lambda_{0}\right)=1$ and then $\lambda \notin \sigma_{p}(A)$.

\section{Step 5:}

There is at most a finite number of eigenvalues of real part in a compact of $\left(0, \lambda_{0}\right)$ and there exists $\varepsilon>0$ such that $\sigma_{p}(A) \backslash\left\{\lambda_{0}\right\} \subset\left\{0<\operatorname{Re} \lambda \leq \lambda_{0}-\varepsilon\right\}$.

Let $\lambda=a+i b$ with $0<a<\lambda_{0}$,

$$
F(\lambda)=1 \text { if and only if }\left\{\begin{array}{l}
\int_{0}^{\infty} \theta(x) e^{-a x} \cos (b x) d x=1 \\
\int_{0}^{\infty} \theta(x) e^{-a x} \sin (b x) d x=0
\end{array}\right.
$$

According to the Riemann-Lebesgue theorem, we have:

$$
\int_{0}^{\infty} \theta(x) e^{-a x} \cos (b x) d x \underset{b \rightarrow+\infty}{\rightarrow} 0
$$

uniformly in $a$ on any compact of $\left\{z \in \mathbb{C} ; \operatorname{Re}(z) \in\left(0, \lambda_{0}\right)\right\}$.

As the eigenvalues are isolated, there can exist only a finite number over each compact of $\left\{0<\operatorname{Re} \lambda<\lambda_{0}\right\}$. Consequently:

$$
\left.\exists \varepsilon>0 ; F(\lambda)=1 \text { and } \lambda \neq \lambda_{0} \Rightarrow \operatorname{Re} \lambda \notin\right] \lambda_{0}-\varepsilon, \lambda_{0}[.
$$

Thus $\sigma_{p}(A) \backslash\left\{\lambda_{0}\right\} \subset\left\{0<\operatorname{Re} \lambda \leq \lambda_{0}-\varepsilon\right\}$.

Proposition 2 (see [2]).

1) The domain $D(A)$ is dense in $L^{1}(1, b)$.

2) $\{\lambda \in \mathbb{C}, \operatorname{Re} \lambda \in] \lambda_{0}-\varepsilon, \lambda_{0}[\} \cup\left\{\lambda \in \mathbb{C} ; \operatorname{Re} \lambda>\lambda_{0}\right\} \subset \rho(A)$, where $\rho(A)$ is the resolvent set of $A$. 
As $D(A)$ is dense in $L^{1}(1, b)$, one can define $\left(A^{*}, D\left(A^{*}\right)\right)$, the adjoint of $(A, D(A))$ :

$$
\begin{aligned}
A^{*} w(x) & =g(x) \partial_{x} w(x)-\beta(x) w(1), \\
D\left(A^{*}\right) & =\left\{w \in L^{\infty}(1, b), g \partial_{x} w \in L^{\infty}(1, b),\right\} .
\end{aligned}
$$

Moreover, one has

Proposition 3. There exists a unique triplet $\left.\left(\lambda_{0}, \phi, V\right) \in\right] 0,+\infty\left[\times D\left(A^{*}\right) \times\right.$ $D(A)$ such that

$$
\left\{\begin{array}{c}
A V=\lambda_{0} V, \quad A^{*} \phi=\lambda_{0} \phi \\
\phi \geq 0, \phi(1)=1, \text { and } \int_{1}^{b} V(x) \phi(x) d x=1 .
\end{array}\right.
$$

More precisely, as $\beta(x)=m x^{\alpha}$ then

$$
\phi \in C^{0}([1, b)), \phi \geq \frac{m}{\lambda_{0}}>0
$$

Proof. We solve $A^{*} \phi=\lambda_{0} \phi$ and get

$$
\phi(x)=e^{\lambda_{0} G(x)}\left(\int_{x}^{b} \frac{\beta(y)}{g(y)} e^{-\lambda_{0} G(y)} d y\right),
$$

with $G$ defined in (12). Recall that $\theta=\beta\left(G^{-1}\right)$, one deduces

$$
\phi(x)=e^{\lambda_{0} G(x)}\left(\int_{G(x)}^{+\infty} \theta(z) e^{-\lambda_{0} z} d z\right)>0 .
$$

So $\phi \in C^{0}([1, b))$. Moreover, as $\beta$ is increasing in $[1, b]$, one has

$$
m x^{\alpha} \frac{e^{-\lambda_{0} G(x)}}{\lambda_{0}} \leq \int_{G(x)}^{+\infty} \theta(z) e^{-\lambda_{0} z} d z \leq m b^{\alpha} \frac{e^{-\lambda_{0} G(x)}}{\lambda_{0}},
$$

so one deduces

$$
\frac{\beta(x)}{\lambda_{0}} \leq \phi(x) \leq \frac{m b^{\alpha}}{\lambda_{0}} .
$$

We finally choose $V(1)$ such that $\int_{1}^{b} V(x) \phi(x) d x=1$.

\subsubsection{The homogeneous problem via a semigroup approach}

Consider

$$
\left\{\begin{array}{l}
\frac{\partial}{\partial t} v(x, t)+\frac{\partial}{\partial x}[g(x) v(x, t)]=0, x \in(1, b), t \geq 0, \\
g(1) v(1, t)=\int_{1}^{b} \beta(x) v(x, t) d x t>0 \\
v(x, 0)=v_{0}(x)
\end{array}\right.
$$


We recall at first some properties about semigroups, let $(X,\|\cdot\|)$ a Banach space (see [8] for more details):

Definition 4. An operator $(A, D(A))$ on a Banach space $X$ is said to be dissipative if:

$$
\|(\lambda I-A) x\| \geq \lambda\|x\|,
$$

Actually we have a more practical criterion in order to establish the dissipativity of an operator. For all $x \in X$, we define the dual set of $x$ by:

$$
J(x)=\left\{x^{\prime} \in X^{\prime} ;\left\langle x, x^{\prime}\right\rangle_{X, X^{\prime}}=\|x\|^{2}=\left\|x^{\prime}\right\|^{2}\right\},
$$

where $X^{\prime}$ is the dual set of $X$ and we recall the result of [8]:

Proposition 5. $(A, D(A))$ is dissipative if and only if for all $x \in D(A)$, there is $j(x) \in J(x)$ such that:

$$
\operatorname{Re}\langle A x, j(x)\rangle_{X, X^{\prime}} \leq 0
$$

Let us show now the main theorem

Theorem 6. The operator $(A, D(A))$ generates a semigroup on $L^{1}(1, b)$.

The theorem 6 is a consequence of the following theorem:

Theorem $7([8])$. Let $(A, D(A))$ a dissipative operator on a Banach space $X$ such that there exists $\lambda>0$ such that $(\lambda I-A)$ is onto, then the restriction of $A$ to $\overline{D(A)}$ generates a contraction semigroup on $\overline{D(A)}$.

In particular:

$$
\left.\begin{array}{c}
\overline{D(A)}=X \\
\forall \lambda>0, R(\lambda I-A)=X \\
\omega \in \mathbb{R} ;(A-\omega I) \text { is dissipative }
\end{array}\right\} \Rightarrow(A, D(A)) \text { generates a semigroup on } X .
$$

The first two points come from Proposition 2. We just have to check the dissipativity condition:

Proposition 8. For all $\omega \geq\|\beta\|_{L^{\infty}}, A-\omega I$ is dissipative.

Proof. Let $v \in D(A)$ and $E=\{x \in(1, b) ; v(x)=0\}$. The function $j(v)=$ $\frac{v}{|v|} \mathbf{1}_{E^{c}}\|v\|_{L^{1}(1, b)}$ belongs to $J(v)$. We have $j(v) \in L^{\infty}$ with $\|j(v)\|_{L^{\infty}} \leq\|v\|_{L^{1}}$. In addition:

$$
\langle v, j(v)\rangle_{L^{1}(1, b), L^{\infty}(1, b)}=\int_{1}^{b} \frac{v^{2}}{|v|} 1_{E^{c}}\|v\|_{L^{1}(1, b)}=\|v\|_{L^{1}(1, b)}^{2}=\|j(v)\|_{L^{\infty}(1, b)}^{2} .
$$


Furthermore,

$$
\begin{aligned}
\langle A v-\omega v, j(v)\rangle_{L^{1}(1, b), L^{\infty}(1, b)} & =-\int_{1}^{b} j(v) \partial_{x}(g v) d x-w \int_{1}^{b} v j(v) d x \\
& =-\int_{1}^{b} j(v) \partial_{x}(g v) d x-w\|v\|_{L^{1}(1, b)}^{2} .
\end{aligned}
$$

We use now lemma 25 proved in the appendix and we get:

$$
\langle A v-\omega v, j(v)\rangle_{L^{1}(1, b), L^{\infty}(1, b)}=g(1)\|v\|_{L^{1}(1, b)}|v(1)|-\omega\|v\|_{L^{1}(1, b)}^{2}
$$

Using the boundary condition $(v \in D(A))$, we get

$$
\begin{aligned}
\langle A v-\omega v, j(v)\rangle_{L^{1}(1, b), L^{\infty}(1, b)} & =\|v\|_{L^{1}(1, b)}\left|\int_{1}^{b} \beta(x) v(x) d x\right|-\omega\|v\|_{L^{1}(1, b)}^{2} \\
& \leq\left(\|\beta\|_{L^{\infty}(1, b)}-\omega\right)\|v\|_{L^{1}(1, b)}^{2} .
\end{aligned}
$$

It is sufficient to choose $\|\beta\|_{L^{\infty}(1, b)} \leq \omega$ in order to obtain (19). Hence we have proved that $(A, D(A))$ generates a semigroup denoted $e^{t A}$.

Let us recall some definitions of semigroup solutions (see [14] for more details).

Definition 9. Let $X$ a Banach space and $(A, D(A))$ a generator of a $C^{0}$ semigroup on $X$, denoted $e^{t A}$. Let

$$
\left\{\begin{array}{l}
y^{\prime}(t)=A y(t), t \in(0, T) \\
y(0)=x
\end{array} .\right.
$$

We call

- weak solution or mild solution of (20), a function $y \in \mathcal{C}([0,+\infty[; X)$ given by

$$
y(t)=e^{t A} x .
$$

- strong solution or classical solution of (20) a function $y \in$ $\mathcal{C}([0,+\infty] ; X) \cap \mathcal{C}^{1}((0,+\infty[; X)$ given by (21) such that $y(t) \in D(A)$ for all $t \in(0,+\infty[$ and satisfying (20) in $[0,+\infty[$.

Moreover one has

Theorem 10. [14] Let $e^{t A}$ a $C_{0}$-semigroup on $X$ of generator $(A, D(A))$. Then:

- for all $x \in X$ (20) has a unique weak solution, 
- for all $x \in D(A)$ (20) has a unique strong solution.

Therefore we have the main result of this subsection :

\section{Theorem 11.}

- For any $v_{0} \in D(A)$ and $T>0$ there exists a unique strong solution $v \in$ $\left.\left.\mathcal{C}([0, T], D(A)) \cap \mathcal{C}^{1}(] 0, T\right], L^{1}(1, b)\right)$. It satisfies the system (18) in $L^{1}(1, b)$

- For any $v_{0} \in L^{1}(1, b)$ and $T>0$, there exists a unique weak solution $v \in \mathcal{C}\left([0, T], L^{1}(1, b)\right)$ such that $(18)$ is verified in the distribution sense.

This ends the existence of solutions of the homogeneous problem associated to (6). In the next subsection we will construct a solution (weak) of (6) for zero initial data. Then we conclude by noticing that the unique solution of (6) is the sum of the homogeneous and this previous.

\subsubsection{The non homogeneous problem via a fixed point argument}

Consider

$$
\left\{\begin{array}{c}
\partial_{t} v+\partial_{x}(g v)=0, x \in(1, b), t>0, \\
g(1) v(1, t)=\int_{1}^{b} \beta(x) v(x, t) d x+f(t), t>0, \\
v(x, 0)=0 .
\end{array}\right.
$$

Definition 12. We call a strong solution on $[0, T]$ of system (22) a function $v \in \mathcal{C}^{1}\left([0, T] ; L^{1}(1, b)\right)$ such that $g v \in \mathcal{C}\left([0, T] ; W^{1,1}(1, b)\right), \lim _{x \rightarrow b^{-}}(g v)(x, t)=0$ for all $t \in[0, T]$ and $v$ satisfies (22). This function $v$ can be viewed as a linear function $\mathcal{T}$ of the source term $f: v=\mathcal{T} f$.

One has

Theorem 13. For any $f \in \mathcal{C}^{1}([0,+\infty[)$ such that $f(0)=0$ there exists a unique strong solution of system (22). Moreover, the operator $\mathcal{T}$ defined on $\left\{f \in \mathcal{C}^{1}([0,+\infty[) ; f(0)=0\}\right.$ satisfies the positivity property:

$$
f \geq 0 \Rightarrow \mathcal{T} f \geq 0 \text {. }
$$

Proof. We are going to construct the strong solution by a fixed point argument. As we have to use a bootstrap argument in time, we have to consider a more general initial data than 0 .

Lemma 14. For any $v_{0} \in L^{1}(1, b)$ such that $g v_{0} \in W^{1,1}(1, b), \lim _{x \rightarrow b^{-}}\left(g v_{0}\right)=0$ and for all function $f \in \mathcal{C}^{1}\left(\left[0,+\infty[)\right.\right.$ such that $f(0)=g(1) v_{0}(1)-\int_{1}^{b} \beta(y) v_{0}(y) d y$, there exists a unique strong solution $v$ of

$$
\left\{\begin{array}{c}
\partial_{t} v+\partial_{x}(g v)=0 \\
g(1) v(1, t)=\int_{1}^{b} \beta(y) v(y, t) d y+f(t) \\
v(., 0)=v_{0}
\end{array}\right.
$$


Proof.

For $0 \leq T_{0}<T$, and $u \in L^{1}(1, b)$ let us define

$$
X_{T}^{T_{0}}(u)=\left\{w \in \mathcal{C}^{1}\left(\left[T_{0}, T\right] ; L^{1}(1, b)\right) ; w\left(., T_{0}\right)=u\right\} .
$$

and

$$
X_{T}^{0}\left(v_{0}\right)=\left\{w \in \mathcal{C}^{1}\left([0, T] ; L^{1}(1, b)\right) ; w(., 0)=v_{0}\right\}=X_{T} .
$$

We endow the set $X_{T}$ with the distance associated by :

$$
\|v\|_{X_{T}}=\sup _{t \in[0, T]}\|v\|_{L^{1}(1, b)}+\sup _{t \in[0, T]}\left\|\partial_{t} v\right\|_{L^{1}(1, b)}
$$

The space $X_{T}$ is a complete metric space. For $w \in X_{T}^{0}\left(v_{0}\right)$, define $\mathcal{T}_{v_{0}, f}(w)=v$ by:

$$
v(x, t)=\left\{\begin{array}{l}
\frac{1}{g(x)}\left(\int_{1}^{b} \beta(y) w(y, t-G(x)) d y+f(t-G(x))\right), G(x)<t \\
\frac{1}{g(x)}\left(g v_{0}\right)\left(G^{-1}(G(x)-t)\right), G(x)>t,
\end{array}\right.
$$

where we recall that $G(x)=\int_{1}^{x} \frac{d y}{g(y)}$. Direct computations show that

$$
\|v(t)\|_{L^{1}(1, b)} \leq t\|\beta\|_{L^{\infty}(1, b)} \sup _{t \in[0, T]}\|w(t)\|_{L^{1}(1, b)}+T \sup _{t \in[0, T]}|f(t)|+\left\|v_{0}\right\|_{L^{1}(1, b)},
$$

and

$$
\lim _{x \rightarrow b^{-}}(g v)(x, t)=0 .
$$

Moreover we observe that $v$ is continuous on $[1, b) \times[0, T] \backslash\{t=G(x)\}$ and from the hypothesis on $w$ we deduce that for all $x_{0} \in[1, b)$ :

$$
\begin{aligned}
\lim _{\substack{G(x)<t \\
(x, t) \rightarrow\left(x_{0}, G\left(x_{0}\right)\right)}}(g v)(x, t) & =\lim _{\substack{G(x)<t \\
(x, t) \rightarrow\left(x_{0}, G\left(x_{0}\right)\right)}}\left(\int_{1}^{b} \beta(y) w(y, t-G(x)) d y+f(t-G(x))\right) \\
& =\left(\int_{1}^{b} \beta(y) w(y, 0) d y+f(0)\right) .
\end{aligned}
$$

By the compatibility conditions satisfied by $v_{0}, f, w(., 0)$, one gets

$$
\lim _{\substack{G(x)<t \\(x, t) \rightarrow\left(x_{0}, G\left(x_{0}\right)\right)}}(g v)(x, t)=\left(g v_{0}\right)(1) .
$$

Besides as $g v_{0} \in W^{1,1}(1, b)$, one has

$$
\begin{aligned}
\lim _{\substack{G(x)<t \\
(x, t) \rightarrow\left(x_{0}, G\left(x_{0}\right)\right)}}(g v)(x, t) & =\lim _{\substack{G(x)<t \\
(x, t) \rightarrow\left(x_{0}, G\left(x_{0}\right)\right)}}\left(g v_{0}\right)\left(G^{-1}(G(x)-t)\right) \\
& =\left(g v_{0}\right)\left(G^{-1}(0)\right) .
\end{aligned}
$$


Recall that $G^{-1}(0)=1$, so one deduces

$$
\lim _{\substack{G(x)>t \\(x, t) \rightarrow\left(x_{0}, G\left(x_{0}\right)\right)}}(g v)(x, t)=\left(g v_{0}\right)(1) .
$$

Therefore $g v \in \mathcal{C}([1, b) \times[0, T])$ and $v(., 0)=v_{0}$. Furthermore, as $w \in X_{T}$ and $g v_{0} \in W^{1,1}(1, b)$, we have for $x<b$

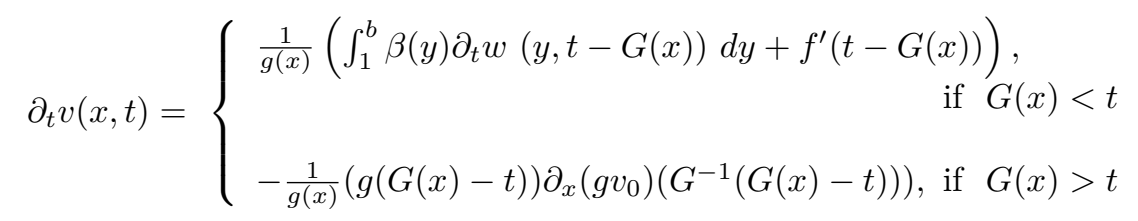

and

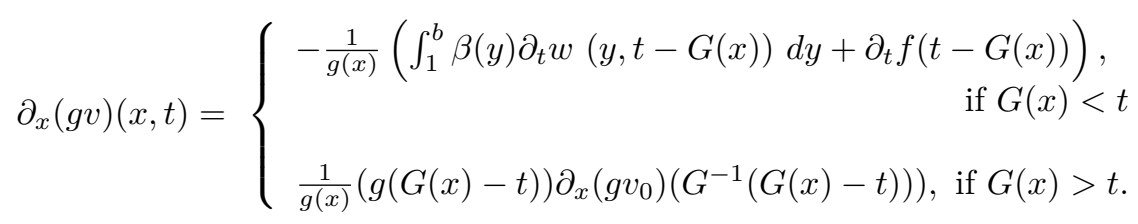

Therefore

$$
\partial_{t} v, \partial_{x}(g v) \in \mathcal{C}\left([0, T] ; L^{1}(1, b)\right)
$$

and

$$
\partial_{t} v+\partial_{x}(g v)=0 .
$$

So $v=\mathcal{T}_{v_{0}, f}(w) \in X_{T}$. Moreover, we remark

$$
\begin{aligned}
\left(\mathcal{T}_{v_{0}, f}\left(w_{1}\right)-\mathcal{T}_{v_{0}, f}\left(w_{2}\right)\right)(x, t) & \\
= & \frac{1}{g(x)}\left(\int_{1}^{b} \beta(y)\left(w_{1}-w_{2}\right)(y, t-G(x)) d y\right) \mathbf{1}_{G(x) \leq t},
\end{aligned}
$$

and

$$
\begin{aligned}
\partial_{t}\left(\mathcal{T}_{v_{0}, f}\left(w_{1}\right)-\mathcal{T}_{v_{0}, f}\left(w_{2}\right)\right)(x, t) & \\
= & \frac{1}{g(x)}\left(\int_{1}^{b} \beta(y) \partial_{t}\left(w_{1}-w_{2}\right)(y, t-G(x)) d y\right) \mathbf{1}_{G(x) \leq t},
\end{aligned}
$$

what gives

$$
\left\|\mathcal{T}_{v_{0}, f}\left(w_{1}\right)-\mathcal{T}_{v_{0}, f}\left(w_{2}\right)\right\|_{X_{T}} \leq T\|\beta\|_{\infty}\left\|w_{1}-w_{2}\right\|_{X_{T}} .
$$

Consequently, for $T<\frac{1}{\|\beta\|_{\infty}}$, the considered application is a contraction on $X_{T}$ and has therefore a unique fixed point. Define $T_{1}=\frac{1}{2\|\beta\|_{\infty}}$. Let us 
denote by $v$ this unique fixed point on $\left[0, T_{1}\right]$. It satisfies $\mathcal{T}_{v_{0}, f}(v)=v$ on $X_{T_{1}}$. One has that $f\left(T_{1}\right)=g(1) v\left(1, T_{1}\right)-\int_{1}^{b}\left(\beta(y) v\left(y, T_{1}\right) d y\right.$, so one can define the operator $\mathcal{T}_{v\left(., T_{1}\right), f}$ on $X_{2 T_{1}}^{T_{1}}\left(v\left(., T_{1}\right)\right)$ and, as $T_{1}$ depends only on $\|\beta\|_{\infty}$, the same arguments show that it has a unique fixed point on $\left[T_{1}, 2 T_{1}\right]$. We can iterate the procedure in any interval of length $T_{1}$ and therefore in $[0,+\infty[$. This ends the proof of the lemma.

The positivity property is a direct consequence of the fact that $w \geq 0$ and, $f \geq 0$ implies that $\mathcal{T}_{0, f}(w) \geq 0$. This ends the proof of the theorem.

Proposition 15. For any $T>0, \mathcal{T}$ is a linear continuous map from the space $\left\{f \in \mathcal{C}^{1}([0, T]) ; f(0)=0\right\}$, endowed with the $L^{1}(0, T)$ norm, to $\mathcal{C}\left([0, T] ; L^{1}(1, b)\right)$.

Proof. By theorem 13, $\mathcal{T}$ is defined from $\left\{f \in \mathcal{C}^{1}([0,+\infty)) ; f(0)=0\right\}$ to $\mathcal{C}\left([0, T], L^{1}(1, b)\right)$ by $\mathcal{T} f=v$ where $v$ is the solution constructed above. Moreover from (24), one can apply lemma 25, and as $v$ satisfies (23), (25), we get that

$$
0=\left\langle\partial_{t} v+\partial_{x}(g v), j(v)\right\rangle_{L^{1}, L^{\infty}} .
$$

thus,

$$
\partial_{t} \int_{1}^{b}|v(x, t)| d x-\left|\int_{1}^{b} \beta(y) v(y, t) d y+f(t)\right|=0
$$

So

$$
\|v(t)\|_{L^{1}(1, b)} \leq\|\beta\|_{L^{\infty}(1, b)} \int_{0}^{t}\|v(s)\|_{L^{1}(1, b)} d s+\int_{0}^{t}|f(s)| d s .
$$

Therefore, by Gronwall lemma, we have

$$
\|v(t)\|_{L^{1}(1, b)} \leq e^{t\|\beta\|_{L^{\infty}(1, b)}} \int_{0}^{T}|f(s)| d s, \forall t \leq T .
$$

Thus one gets

$$
\|\mathcal{T} f\|_{\mathcal{C}\left([0, T] ; L^{1}(1, b)\right)} \leq e^{T\|\beta\|_{L^{\infty}(1, b)}\|f\|_{L^{1}(0, T)}}
$$

Now we are able to construct a weak solution of system (22).

Definition 16. We say that $v$ is a weak solution of system (22) if there exists a sequence $\left(f_{n} \subset \mathcal{C}^{1}([0,+\infty))\right.$ such that $f_{n}(0)=0$ that converges to $f$ in $L^{1}(0, T)$ for any $T>0$ and $\left(\mathcal{T} f_{n}\right)$ converges to $v$ in $\mathcal{C}\left([0, T] ; L^{1}(1, b)\right)$.

Notice that by (26) the limit is independently of the choice of the sequence $\left(f_{n}\right)$. Therefore by proposition 15 , one has 
Proposition 17. For any $f \in L_{l o c}^{1}(0,+\infty)$ there exists a unique weak solution. We still denote $\mathcal{T}$ the extension of $\mathcal{T}$ to $L_{\text {loc }}^{1}(0,+\infty)$.

Proof. It suffices to notice that if $f \in L_{l o c}^{1}(0,+\infty)$ there exists a sequence $f_{n} \in \mathcal{C}^{1}\left(\left[0,+\infty[)\right.\right.$ such that $f_{n}(0)=0$ that converges toward $f$ in $L_{l o c}^{1}(0,+\infty)$ and to use (26).

\subsubsection{Application to Von Foerster equation with Gompertzian growth}

From the last subsections one can deduce an existence and uniqueness result for the global problem (6) :

Theorem 18. For any $v_{0} \in L^{1}(1, b)$ and $f \in \mathcal{C}([0,+\infty[)$, there exists an unique weak solution $v \in \mathcal{C}\left(\left[0,+\infty\left[; L^{1}(1, b)\right)\right.\right.$ of system (6) given by

$$
v(t)=e^{t A} v_{0}+\mathcal{T} f(t) .
$$

Moreover if $v_{0} \in D(A)$ and if $f \in \mathcal{C}^{1}([0,+\infty[)$ such that $f(0)=0$, there exists a unique strong solution $v$, still given by (27), such that $f(t)=g(1) v(1, t)-$ $\int_{1}^{b} \beta(y) v(y, t) d y$, for all $t \in[0,+\infty[$.

\subsection{Asymptotic behaviour of weak solutions}

\subsubsection{The homogeneous problem}

We start considering the homogeneous problem (18). What follows is an adaptation of ideas that can be found in $[15],[12]$. Let us decompose the space $L^{1}(1, b)$ into :

$$
L^{1}(1, b)=\mathbb{R} V \oplus(\mathbb{R} \phi)^{\perp},
$$

where

$$
(\mathbb{R} \phi)^{\perp}=\left\{u \in L^{1}(1, b),\langle u, \phi\rangle_{L^{1}, L^{\infty}}=\int_{1}^{b} u \phi=0\right\} .
$$

and $\phi$ is the eigenfunction defined in proposition 3. Any $u \in L^{1}(1, b)$, can be decomposed into

$$
u=\langle u, \phi\rangle_{L^{1}, L^{\infty}} V+\left(u-\langle u, \phi\rangle_{L^{1}, L^{\infty}} V\right)
$$

Actually let $L_{\phi}^{1}(1, b)$ be the space:

$$
L_{\phi}^{1}(1, b)=L^{1}((1, b) ; \phi d x)
$$

endowed with the norm $\|u\|_{\phi}:=\int_{1}^{b}|u(x)| \phi(x) d x$. Thanks to the proposition $3,\|\cdot\|_{\phi}$ defines a norm equivalent to the $L^{1}$ norm. We state now an important proposition giving some properties of the weak solutions: 
Theorem 19. For any initial data in $L^{1}(1, b)$, the weak solution of the homogeneous problem (18) satisfies

1) Decreasing of the norm $L_{\phi}^{1}(1, b)$

$$
\left\|e^{-\lambda_{0} t} v(t)\right\|_{L_{\phi}^{1}(1, b)} \leq\left\|v_{0}\right\|_{L_{\phi}^{1}(1, b)} .
$$

2) Conservation of the mean value in $L_{\phi}^{1}(1, b)$

$$
\int_{1}^{b} e^{-\lambda_{0} t} v(t) \phi(x) d x=\int_{1}^{b} v_{0}(x) \phi(x) d x, \forall t \geq 0
$$

3) Comparison principle

$$
v_{0,1} \leq v_{0,2} \Rightarrow v_{1}(t) \leq v_{2}(t)
$$

Proof. Let $v_{0} \in D(A)$ and $\widetilde{v}(t)=e^{-\lambda_{0} t} v(t)$, we have

$$
\left\{\begin{array}{c}
\partial_{t} \widetilde{v}+\partial_{x}(g(x) \widetilde{v})+\lambda_{0} \widetilde{v}=0, x \in(1, b), t>0, \\
g(1) \widetilde{v}(1, t)=\int_{1}^{b} \beta(x) \widetilde{v}(x, t) d x, t>0, \\
\widetilde{v}(x, 0)=v_{0}(x) .
\end{array}\right.
$$

But $\phi$ satisfies

$$
\left\{\begin{array}{c}
-g(x) \partial_{x} \phi(x)+\lambda_{0} \phi(x)=\beta(x), x \in(1, b) \\
\phi>0, \phi(1)=1, \text { and } \int_{1}^{b} V(x) \phi(x) d x=1
\end{array}\right.
$$

Therefore we have

$$
\partial_{t}(\widetilde{v} \phi)(x, t)+\partial_{x}(g \widetilde{v} \phi)(x, t)=-\beta \widetilde{v}(x, t), x \in(1, b), t>0 .
$$

One can remark that $\tilde{v} \in \mathcal{C}\left(\left[0,+\infty[; D(A)) \cap \mathcal{C}^{1}\left(\left[0,+\infty\left[; L^{1}(1, b)\right)\right.\right.\right.\right.$ and so as for $\widetilde{v} \phi$. Using this fact, and Lemma 25 we obtain

$$
\frac{d}{d t} \int_{1}^{b}|\widetilde{v}| \phi d x+\int_{1}^{b} \partial_{x}(g|\widetilde{v} \phi|) d x=-\int_{1}^{b} \beta|\widetilde{v}(x, t)| d x, t>0 .
$$

Integrating by parts and taking into account the boundary condition on $\widetilde{v}$, we get

$$
\frac{d}{d t} \int_{1}^{b}|\widetilde{v}| \phi d x-\left|\int_{1}^{b} \beta \widetilde{v}(x, t) d x\right|=-\int_{1}^{b} \beta|\widetilde{v}(x, t)| d x, t>0
$$

hence

$$
\frac{d}{d t} \int_{1}^{b}|\widetilde{v}| \phi d x=\left|\int_{1}^{b} \beta \widetilde{v} d x\right|-\int_{1}^{b} \beta|\widetilde{v}| d x \leq 0, \text { as } \beta>0 .
$$


Integrating in time we get:

$$
\left\|e^{-\lambda_{0} t} v(t)\right\|_{L_{\phi}^{1}(1, b)} \leq\left\|v_{0}\right\|_{L_{\phi}^{1}(1, b)}, \forall t \geq 0, \forall v_{0} \in L^{1}(1, b) .
$$

Hence the inequality (28) follows immediately for any $v_{0} \in D(A)$. By density of $D(A)$ in $L^{1}(1, b)$ we deduce that (28) holds for any initial data in $L^{1}(1, b)$. In order to get $(29)$ one integrates $(30)$ with respect to $x$

$$
\frac{d}{d t} \int_{1}^{b} \widetilde{v} \phi d x+\int_{1}^{b} \partial_{x}(\widetilde{v} \phi g) d x=-\int_{1}^{b} \beta \widetilde{v}(x, t) d x .
$$

Using the boundary condition, we finally obtain:

$$
\frac{d}{d t} \int_{1}^{b} \tilde{v} \phi d x=0
$$

Let us show the positivity property:

We start again from the equation (30). We first prove that if $v_{0} \leq 0$ then $v(t) \leq 0, \forall t \geq 0$, for $v_{0} \in D(A)$. Let us consider the function

$$
\operatorname{sgn}^{+}(v)=\frac{\max (v, 0)}{|v|} \mathbf{1}_{E^{c}}
$$

As in lemma 25 one gets:

$$
\int_{1}^{b} \partial_{x}(g v) \operatorname{sgn}^{+}(v) d x=-g(1)\|v\|_{L^{1}(1, b)} v_{+}(1)
$$

We multiply now the equation $(30)$ by $\operatorname{sgn}^{+}(\tilde{v})$ and we obtain:

$$
\frac{d}{d t} \int_{1}^{b} \tilde{v}^{+} \phi d x \leq 0
$$

integrating in time we have

$$
\int_{1}^{b} \tilde{v}^{+}(x, t) \phi(x) d x \leq \int_{1}^{b} v^{+}(x, 0) \phi(x) d x,
$$

and as $v^{+}(x, 0)=0$, we derive

$$
\int_{1}^{b} \tilde{v}^{+} \phi d x=0, \forall t \geq 0
$$

As we have already shown that $\phi \geq \frac{m b^{\alpha}}{\lambda_{0}}$, one gets

$$
\tilde{v}^{+}(x, t)=0, \forall t \geq 0, \text { or } v(t) \leq 0 .
$$

Thus we have proved the monotony property for all initial data in $D(A)$ and then by density for any initial data in $L^{1}(1, b)$. 
We state now our principal result concerning the asymptotic behaviour in the case of the model. As in [15], one has

Theorem 20. There exists $\gamma>0$ such that $\beta(x) \geq \gamma \phi(x), \forall x \in[1, b]$. Moreover for any $v_{0} \in(\mathbb{R} \phi)^{\perp}$, one has

$$
\left\|e^{-\lambda_{0} t} e^{t A} v_{0}\right\|_{L_{\phi}^{1}(1, b)} \leq e^{-\gamma t}\left\|v_{0}\right\|_{L_{\phi}^{1}(1, b)} .
$$

Therefore for any $v_{0} \in L^{1}(1, b)$ we have:

$$
\lim _{t \rightarrow+\infty} e^{\left(-\lambda_{0}+\gamma^{\prime}\right) t}\left\|e^{t A} v_{0}-e^{\lambda_{0} t}\left\langle v_{0}, \phi\right\rangle_{L^{1}, L^{\infty}} V\right\|_{L_{\phi}^{1}(1, b)}=0, \forall \gamma^{\prime}<\gamma
$$

Proof. From (17)) one can verify that $\gamma \geq \frac{\lambda_{0}}{b^{\alpha}}$. Let $v_{0} \in(\mathbb{R} \phi)^{\perp}$, as $e^{-\lambda_{0} t} e^{t A} v_{0}$ has zero mean value in $L_{\phi}^{1}(1, b)$, from (31) one can deduce

$$
\begin{aligned}
\frac{d}{d t} \int_{1}^{b}\left|e^{-\lambda_{0} t} e^{t A} v_{0}\right| \phi d x & =\left|\int_{1}^{b} \beta e^{-\lambda_{0} t} e^{t A} v_{0} d x\right|-\int_{1}^{b} \beta\left|e^{-\lambda_{0} t} e^{t A} v_{0}\right| d x \\
& =\left|\int_{1}^{b}(\beta-\gamma \phi) e^{-\lambda_{0} t} e^{t A} v_{0} d x\right|-\int_{1}^{b} \beta\left|e^{-\lambda_{0} t} e^{t A} v_{0}\right| d x \\
& \leq \int_{1}^{b}\left|(\beta-\gamma \phi) e^{-\lambda_{0} t} e^{t A} v_{0}\right| d x-\int_{1}^{b} \beta\left|e^{-\lambda_{0} t} e^{t A} v_{0}\right| d x \\
& \leq \int_{1}^{b}(\beta-\gamma \phi)\left|e^{-\lambda_{0} t} e^{t A} v_{0}\right| d x-\int_{1}^{b} \beta\left|e^{-\lambda_{0} t} e^{t A} v_{0}\right| d x \\
& \leq-\gamma \int_{1}^{b}\left|e^{-\lambda_{0} t} e^{t A} v_{0}\right| \phi d x,
\end{aligned}
$$

therefore

$$
\int_{1}^{b}\left|e^{-\lambda_{0} t} e^{t A} v_{0}\right| \phi d x \leq e^{-\gamma t} \int_{1}^{b}\left|v_{0}\right| \phi d x
$$

As for all $v_{0} \in L^{1}(1, b)$

$$
v_{0}=\left\langle v_{0}, \phi\right\rangle_{L^{1}, L^{\infty}} V+\left(v_{0}-\left\langle v_{0}, \phi\right\rangle_{L^{1}, L^{\infty}} V\right),
$$

one gets

$$
e^{t A} v_{0}=\left\langle v_{0}, \phi\right\rangle_{L^{1}, L^{\infty}} e^{\lambda_{0} t} V+e^{t A}\left(v_{0}-\left\langle v_{0}, \phi\right\rangle_{L^{1}, L^{\infty}} V\right) .
$$

Then we have:

$$
e^{-\lambda_{0} t} e^{t A} v_{0}=\left\langle v_{0}, \phi\right\rangle_{L^{1}, L^{\infty}} V+e^{-\lambda_{0} t} e^{t A}\left(v_{0}-\left\langle v_{0}, \phi\right\rangle_{L^{1}, L^{\infty}} V\right) .
$$


Therefore, taking the norm and using (32), we obtain

$$
\left\|e^{-\lambda_{0} t} e^{t A} v_{0}-\left\langle v_{0}, \phi\right\rangle_{L^{1}, L^{\infty}} V\right\|_{L_{\phi}^{1}(1, b)} \leq e^{-\gamma t}\left\|v_{0}-\left\langle v_{0}, \phi\right\rangle_{L^{1}, L^{\infty}} V\right\|_{L_{\phi}^{1}(1, b)}
$$

which implies that

$$
e^{-\lambda_{0} t}\left\|e^{t A} v_{0}-e^{\lambda_{0} t}\left\langle v_{0}, \phi\right\rangle V\right\|_{L_{\phi}^{1}(1, b)} \leq e^{-\gamma t}\left\|v_{0}-\left\langle v_{0}, \phi\right\rangle_{L^{1}, L^{\infty}} V\right\|_{L_{\phi}^{1}(1, b)}
$$

and concludes the proof of the theorem.

\subsubsection{The non homogeneous problem}

We focus now on the asymptotic behaviour of the weak solutions for the non homogeneous problem.

Theorem 21. For all initial data $v_{0} \in L^{1}(1, b)$ and $f \in \mathcal{C}([0,+\infty[)$ the unique weak solution of (22) satisfies for all $t \geq 0$ :

i)

$$
\int_{1}^{b} e^{-\lambda_{0} t} \mathcal{T} f(x, t) \phi(x) d x=\int_{0}^{t} e^{-\lambda_{0} \tau} f(\tau) d \tau
$$

ii)

$$
\|\mathcal{T} f(t)\|_{L_{\phi}^{1}(1, b)} \leq e^{\lambda_{0} t} \int_{0}^{t} e^{-\lambda_{0} \tau}|f(\tau)| d \tau, \forall t \geq 0
$$

Proof. Consider $f_{n} \in\left\{\mathcal{C}^{1}\left[0,+\infty\left[; f_{n}(0)=0\right\}\right.\right.$ such that $f_{n} \rightarrow f$ in $L_{l o c}^{1}(0,+\infty)$. From (24), one has

$$
\frac{d}{d t}\left(\int_{1}^{b} e^{-\lambda_{0} t} \mathcal{T} f_{n}(x, t) \phi(x) d x-\int_{0}^{t} e^{-\lambda_{0} \tau} f_{n}(\tau) d \tau\right)=0 .
$$

Integrating in time, passing to the limit and taking into account the continuity of $\mathcal{T}$, we deduce the first item. Besides, still by (24) one can apply lemma 25 to $e^{\lambda_{0} t} \mathcal{T} f_{n}$ :

$$
\int_{1}^{b} \phi e^{-\lambda_{0} t}\left|\mathcal{T} f_{n}(x, t)\right| d x \leq \int_{0}^{t} e^{-\lambda_{0} \tau}\left|f_{n}(\tau)\right| d \tau
$$

Once again, passing to the limit we obtain the second item. This ends the proof of the theorem.

We can deduce now the principal result:

\section{Theorem 22 (Asymptotic behaviour of weak solutions).}

i) For any $v_{0} \in(\mathbb{R} \phi)^{\perp}$, the unique weak solution of (22) verifies

$$
e^{-\lambda_{0} t}\|v(t)\|_{L_{\phi}^{1}(1, b)} \leq \int_{0}^{t} e^{-\lambda_{0} \tau}|f(\tau)| d \tau
$$


ii) There exists $\gamma>0$ such that for any $v_{0} \in L_{\phi}^{1}(1, b)$

$$
\left\|e^{-\lambda_{0} t} v(t)-\left\langle v_{0}, \phi\right\rangle_{L^{1}, L^{\infty}} V\right\|_{L_{\phi}^{1}(1, b)} \leq e^{-\gamma t}\left\|v_{0}\right\|_{L_{\phi}^{1}(1, b)}+\int_{0}^{t} e^{-\lambda_{0} \tau}|f(\tau)| d \tau .
$$

Proof. Recall that $v(t)=e^{t A} v_{0}+\mathcal{T} f(., t)$. From theorem 20 and theorem 21, one deduces the two items.

\subsubsection{Application to Von Foerster equation with Gompertzian growth}

In the model of growing metastatic tumors we have $v_{0}=0$ and $f>0$, the unique solution of (1) is nonnegative and we have:

$$
\|v(t)\|_{L_{\phi}^{1}(1, b)}=e^{\lambda_{0} t} \int_{0}^{t} e^{-\lambda_{0} \tau} f(\tau) d \tau=e^{\lambda_{0} t} \int_{0}^{t} e^{-\lambda_{0} \tau} \beta\left(x_{p}(\tau)\right) d \tau .
$$

We define now an interesting quantity for clinical applications. For a clinician, a useful data to know is without a doubt the total number $N_{1}$ of metastases and in particular the number of large size metastases that is to say for example the tumors whose size is larger than $10^{9}$ cells, which correspond approximately to a tumor of $30 \mathrm{~g}$.

$$
N_{b_{\min }}(t)=\int_{b_{\min }}^{b} v(x, t) d x
$$

We can give an estimate of $N_{1}$. By (17) we have $\frac{m}{\lambda_{0}} \leq \phi \leq \frac{m b^{\alpha}}{\lambda_{0}}$. Therefore, as

$$
\frac{1}{\max \phi}\|v\|_{L_{\phi}^{1}} \leq N_{1}(t)=\int_{1}^{b} \frac{1}{\phi(x)} v(x, t) \phi(x) d x \leq \frac{1}{\min \phi}\|v\|_{L_{\phi}^{1}}
$$

we get

$$
\lambda_{0} e^{\lambda_{0} t} \int_{0}^{t} e^{-\lambda_{0} \tau} b^{-\alpha e^{-a \tau}} d \tau \leq N_{1}(t) \leq \lambda_{0} b^{\alpha} e^{\lambda_{0} t} \int_{0}^{t} e^{-\lambda_{0} \tau} b^{-\alpha e^{-a \tau}} d \tau
$$

We derive that for all $t \geq 0$,

$$
m b^{-1} e^{\lambda_{0} t}-1 \leq N_{1}(t) \leq b^{\alpha} e^{\lambda_{0} t} .
$$

We can bring this to light in next section, with the numerical simulations.

An interesting fact to observe is that if we neglect the effect of the growth due to the metastases itself for small times, that is to say:

$$
\left\{\begin{array}{l}
\frac{\partial}{\partial t} \rho(x, t)+\frac{\partial}{\partial x}(g(x) \rho(x, t))=0, \forall x \in[1, b], \forall t \geq 0 \\
g(1) \rho(1, t)=\beta\left(x_{p}(t)\right), \forall t \geq 0 \\
\rho(0, x)=0, \forall x \in[1, b]
\end{array}\right.
$$


one obtains:

$$
N_{\rho}(t):=\int_{1}^{b} v(x, t) d x=\int_{0}^{t} \beta\left(x_{p}(\tau)\right) d \tau=m \int_{0}^{t} b^{\alpha}\left(\frac{1}{b}\right)^{\alpha e^{-a \tau}} d \tau .
$$

We will see in the numerical simulations that $N_{1}(t)$ does not exceed 1 while more than one year and that the influence of the emission of metastases by metastases itself is also long to appear.

\section{Numerical analysis}

We propose in this section a numerical approximation of problem (1) for data coming from clinical observations of a metastatic hepatocarcinoma, (see [11]):

$$
a=0.00286, b=7.3 \times 10^{10}, \alpha=\frac{2}{3}, m=5.3 \times 10^{-8} .
$$

\subsection{A characteristics scheme}

Due to various scales imposed by the clinical data, we observed that classical upwind finite volume schemes require a very fine discretization in order to be stable for long time. That is why we prefered to use a different approach using the behaviour of the solution of (1) along the characteristic curves. Such methods have been investigated in [1] for more general growth speed $g$ but for space intervals of length 1 . In the case of Gompertz growth, the scheme derivation is made easier, by the explicit expression of the solutions along the characteristics (2).

Let $k$ be a constant time step discretization, $k=\frac{T}{N}$ on this interval $[0, T]$ :

$$
0=t_{1}<\cdots<t_{n}=(n-1) k<\cdots<T_{N+1}=T .
$$

The spatial discretization can be restricted to the interval $\left[1, x_{p}(T)\right]$, since we saw in the section 3.1.3 that the solution vanishes for $(t, x), x \geq x_{p}(T)$. Let $x_{n}=x_{p}\left(t_{n}\right)$ this discretization:

$$
1=x_{1}<x_{2}<\cdots<x_{N}<x_{N+1}=x_{p}(T) .
$$

We then denote by $v_{i}^{n}$ an approximation of the solution $v$ of problem (1) at the point $\left(x_{i}, t_{n}\right)$.

The key point of the construction of this scheme is that the points $\left(x_{i}, t_{n}\right)$ and $\left(x_{i+1}, t_{n+1}\right)$ belong to the same characteristic for any $n \geq 1$ and $i \geq$ 1. For all $n \geq 1$, we solve explicitely the transport equation on the time interval $\left[t_{n}, t_{n+1}\right]$ and we use a first order quadrature method to take into 
account the boundary condition. Computing the characteristics between the point $\left(t_{n}, x_{i-1}\right)$ and $\left(t_{n+1}, x_{i}\right)$, we obtain the following scheme:

$$
\left\{\begin{array}{l}
v_{1}^{0}=\frac{1}{g(1)} \beta\left(x_{p}(0)\right), v_{i}^{0}=0,2 \leq i \leq N+1 \\
v_{i}^{n+1}=v_{i-1}^{n} e^{a k} f_{i-1}(k), i=2, \cdots, N+1, n=1, \cdots, N \\
v_{1}^{n+1}=\frac{1}{g(1)} \beta\left(x_{p}\left(t_{n+1}\right)\right)+\frac{1}{g(1)} \sum_{i=2}^{N} h_{i} \beta\left(x_{i}\right) v_{i}^{n+1}, n=1, \cdots, N
\end{array}\right.
$$

where $f_{i}(k)=\left(\frac{x_{i}}{b}\right)^{1-e^{-a k}}$ and $h_{i}=x_{i}-x_{i-1}$.

Note that $v_{i}^{n}=0$ for all $i=n+2, \ldots, N+1$.

\subsection{Convergence analysis}

We prove in this section the convergence of the characteristics scheme. We denote $e_{i}^{n}=v\left(x_{i}, t_{n}\right)-v_{i}^{n}$ the error of the scheme. Remarking that if one starts from the exact solution at the points $\left(x_{i}, t_{n}\right)$ then the scheme is exact at the points $\left(x_{i}, t_{n+1}\right)$ :

$$
v\left(x_{i}, t_{n+1}\right)=v\left(x_{i-1}, t_{n}\right) f_{i-1}(k) e^{a k}
$$

we derive

$$
\left\{\begin{array}{l}
e_{i}^{n+1}=e_{i-1}^{n} f_{i-1}(k) e^{a k} \\
e_{1}^{n+1}=\frac{1}{g(1)} \sum_{i=2}^{N} h_{i} \beta_{i} e_{i}^{n+1}+r^{n+1} \\
e_{i}^{0}=0
\end{array}\right.
$$

with $r^{n}=\frac{1}{g(1)}\left(\int_{1}^{b} \beta(x) v\left(x, t_{n}\right) d x-\sum_{i=2}^{N} h_{i} \beta\left(x_{i}\right) v\left(x_{i}, t_{n}\right)\right)$. The truncation error of the quadrature $r^{n}$ only comes from the quadrature error; one can estimate $r^{n}$ as soon as the function $\beta v$ is smooth:

Proposition 23 (Truncation error). Let $\Omega_{T}=\left\{(x, t) \in[1, b) \times\left[0, \frac{T}{a}\right] ; x \leq\right.$ $\left.x_{p}(t)\right\}$ and $v \in \mathcal{C}^{1}\left(\Omega_{T}\right)$. There exists $C_{T}=C(T, b)$ such that

$$
\left|r^{n}\right| \leq k C_{T} \sup _{\left[1, x_{p}\left(t_{n}\right)\right]}\left|\partial_{x}(\beta v)\left(\ldots, t_{n}\right)\right|, \forall t_{n} \leq \frac{T}{a} .
$$


Proof. Using the fact that $v\left(x, t_{n}\right)=0$ for all $x>x_{p}\left(t_{n}\right)$, we can write that

$$
\begin{aligned}
r^{n} & =\frac{1}{g(1)}\left(\sum_{i=2}^{n+1} \int_{x_{i-1}}^{x_{i}}\left(\beta(x) v\left(x, t_{n}\right)-\beta\left(x_{i}\right) v\left(x_{i}, t_{n}\right)\right) d x\right) \\
& =\frac{1}{g(1)}\left(\sum_{i=2}^{n+1} \int_{x_{i-1}}^{x_{i}} \int_{x_{i-1}}^{x} \partial_{x}\left(\beta v\left(\cdot, t_{n}\right)\right)(s) d s d x\right) \\
& \leq \frac{\sup _{\left[0, x_{p}\left(t_{n}\right)\right]}\left|\partial_{x}\left(\beta v\left(\cdot, t_{n}\right)\right)\right|}{2 g(1)}\left(\sum_{i=2}^{n+1}\left(x_{i}-x_{i-1}\right)^{2}\right)
\end{aligned}
$$

Let us define the function $\Psi(x)=x\left(1-\left(\frac{b}{x}\right)^{1-e^{a k}}\right)$ and remark that $x_{i}-$ $x_{i-1}=\Psi\left(x_{i}\right)$. We derive that

$$
\sum_{i=2}^{n+1}\left(x_{i}-x_{i-1}\right)^{2}=\sum_{i=2}^{n+1}\left(x_{i}-x_{i-1}\right) \Psi\left(x_{i-1}\right) .
$$

The function $\Psi$ is a concave on $[1, b]$, it increases on $\left[1, x^{*}\right]$ with $x^{*}=b e^{-\frac{a k}{e^{a k}-1}}$ and decreases on $\left[x^{*}, b\right]$.

While $x_{p}\left(t_{n+1}\right)<x^{*}$, that is while $a t_{n+1}<\ln (\ln (b))$,

$$
\begin{aligned}
\sum_{i=2}^{n+1}\left(x_{i}-x_{i-1}\right)^{2} & \leq\left(x_{p}\left(t_{n+1}\right)-1\right) \Psi\left(x_{p}(t)\right) \\
& \leq\left(b^{1-e^{-a t_{n}}}-1\right) b^{1-e^{-a t_{n}}}\left(1-b^{e^{-a t_{n}}\left(1-e^{a k}\right)}\right) \\
& \leq 2\left(b^{1-e^{-T}}-1\right) b^{1-e^{-T}} e^{-T} a k \ln (b)
\end{aligned}
$$

If $a t_{n+1} \geq \ln (\ln (b))$, the estimate becomes

$$
\sum_{i=2}^{n+1}\left(x_{i}-x_{i-1}\right)^{2} \leq 2\left(x_{p}\left(t_{n}\right)-1\right) \Psi\left(x^{*}\right) \leq\left(b^{1-e^{-a t_{n}}}-1\right) b a k .
$$

This conclude the proof with $C_{T}=\left(b^{1-e^{-T}}-1\right) b^{1-e^{-T}} e^{-T}$ if $T<\ln (\ln (b))$ and $C_{T}=\frac{b}{\ln (b)}\left(b^{1-e^{-T}}-1\right)$ else.

The convergence of the scheme is a consequence of the following error estimates:

Theorem 24 (Error estimates). Let $\Omega_{T}=\left\{(x, t) \in[1, b) \times\left[0, \frac{T}{a}\right] ; x \leq x_{p}(t)\right\}$ and $v \in \mathcal{C}^{1}\left(\Omega_{T}\right)$. 
1) There exists $C_{T}=C(T, \beta, v, b)$ and $k_{0}$ such that for all $k \leq k_{0}$

$$
\left\|e^{n}\right\|_{1} \stackrel{\text { def }}{=} \sum_{i=1}^{N} h_{i}\left|e_{i}^{n}\right| \leq C_{T} k e^{\lambda_{0} t_{n}} .
$$

2) There exists $C_{T}=C(T, \beta, v)$ such that

$$
\left\|e^{n}\right\|_{1, \phi} \stackrel{\text { def }}{=} \sum_{i=1}^{N} h_{i}\left|e_{i}^{n} \phi\left(x_{i}\right)\right| \leq C_{T} k e^{\lambda_{0} t_{n}} .
$$

Note that strong solutions of the problem (1) fullfills the assumptions of this theorem and thus the convergence of the characteristic scheme (35) is proved for the strong solutions of problem (1) for long time intervals.

Proof.

1) Error estimate in $L^{1}$ norm

We have

$$
\begin{aligned}
\left\|e^{n+1}\right\|_{1} & =h_{1}\left|e_{1}^{n+1}\right|+e^{a k} \sum_{i=2}^{N} h_{i}\left|e_{i-1}^{n}\right| f_{i-1}(k) \\
& =h_{1}\left|e_{1}^{n+1}\right|+e^{a k} \sum_{i=1}^{N-1} h_{i+1}\left|e_{i}^{n}\right| f_{i}(k)
\end{aligned}
$$

with

$$
\begin{aligned}
h_{1}\left|e_{1}^{n+1}\right| & \leq \frac{h_{1}}{a \ln b} \sum_{i=2}^{N} h_{i} \beta_{i}\left|e_{i}^{n+1}\right|+h_{1}\left|r^{n+1}\right| \\
& \leq \frac{h_{1}}{a \ln b} \sum_{i=1}^{N-1} h_{i+1} \beta_{i+1} e^{a k} f_{i}(k)\left|e_{i}^{n}\right|+h_{1}\left|r^{n+1}\right| .
\end{aligned}
$$

Hence we obtain

$$
\left\|e^{n+1}\right\|_{1} \leq h_{1}\left|r^{n+1}\right|+\sum_{i=1}^{N-1} h_{i}\left|e_{i}^{n}\right| e^{a k} A_{i}^{1} A_{i}^{2} .
$$

with $A_{i}^{1}=f_{i}(k) \frac{h_{i+1}}{h_{i}}$ and $A_{i}^{2}=\left(1+h_{1} \frac{\beta_{i+1}}{a \ln b}\right)$. Remark that:

- For any $k>0$ and any $i \geq 1, A_{i}^{1} \leq 1$.

Indeed, we can write

$$
\begin{aligned}
h_{i} & =e^{\ln (b)\left(1-e^{-a i k}\right)}\left(1-e^{\ln (b) e^{-a i k}\left(1-e^{a k}\right)}\right) \\
h_{i+1} & =e^{\ln (b)\left(1-e^{-a i k}\right)}\left(e^{\ln (b) e^{-a i k}\left(1-e^{-a k}\right)}-1\right)
\end{aligned}
$$


Hence,

$$
\begin{aligned}
f_{i}(k) \frac{h_{i+1}}{h_{i}} & =e^{-\ln (b) e^{-i a k}\left(1-e^{-a k}\right)} \frac{e^{\ln (b) e^{-a i k}\left(1-e^{-a k}\right)}-1}{1-e^{\ln (b) e^{-a i k}\left(1-e^{a k}\right)}} \\
& =\frac{1-z^{e^{-a k}}}{1-z} \leq 1
\end{aligned}
$$

as $z=e^{-\ln (b) e^{-a i k}\left(e^{a k}-1\right)} \leq 1$ and then $1-z^{e^{-a k}} \leq 1-z$.

- There exists $k_{0}$ such that for all $k \leq k_{0}, A_{i}^{2} \leq 1+2 k m b^{\alpha} \leq e^{2 k m b^{\alpha}}$.

This is indeed a corollary of the mean value theorem:

$$
h_{1}=x_{p}(k)-1=b^{1-e^{-a k}}-1 \leq a k \ln (b) \sup _{x \in[0, a k]}\left(e^{-x} e^{\ln (b)\left(1-e^{-x}\right)}\right) \leq 2 a k \ln (b)
$$

as soon as $k \leq k_{0}$ where $k_{0}=\frac{1}{a} \ln \left(\frac{1}{1-\frac{\ln (2)}{\ln (b)}}\right) \sim 0.98$.

The estimate $(36)$ becomes for $k \leq k_{0}$ :

$$
\left\|e^{n+1}\right\|_{1} \leq 2 a k \ln (b) r_{n+1}+\left\|e^{n}\right\|_{1} e^{k\left(a+2 m b^{\alpha}\right)} .
$$

Using a discrete Gronwall lemma we get:

$$
\left\|e^{n}\right\|_{1} \leq 2 a k \ln (b) \sum_{l=0}^{n-1} r_{l} e^{(n-1-l) k\left(a+2 m b^{\alpha}\right)}
$$

so that

$$
\left\|e^{n}\right\|_{1} \leq 2 a k \ln (b) \max _{l=1, \ldots, n} r_{l} \frac{e^{\left(a+2 m b^{\alpha}\right) t_{n}}-1}{e^{\left(a+2 m b^{\alpha}\right) k}-1}
$$

We conclude using Proposition 23 and the fact that $\underbrace{2 m b^{\alpha}}_{\sim 1.851488869} \leq \lambda_{0}$.

\section{2) Error estimate in $L_{\phi}^{1}$ norm}

Now we have

$$
\begin{aligned}
\left\|e^{n+1}\right\|_{1, \phi} & =h_{1} \phi\left(x_{1}\right)\left|e_{1}^{n+1}\right|+e^{a k} \sum_{i=2}^{N} h_{i} \phi\left(x_{i}\right)\left|e_{i-1}^{n}\right| f_{i-1}(k) \\
& =h_{1} \phi(1)\left|e_{1}^{n+1}\right|+e^{a k} \sum_{i=1}^{N-1} h_{i+1} \phi\left(x_{i+1}\right)\left|e_{i}^{n}\right| f_{i}(k)
\end{aligned}
$$


with as $\phi(1)=1$

$$
\begin{aligned}
h_{1} \phi(1)\left|e_{1}^{n+1}\right| & \leq \frac{h_{1}}{g(1)} \sum_{i=2}^{N} h_{i} \beta_{i}\left|e_{i}^{n+1}\right|+h_{1}\left|r^{n+1}\right| \\
& \leq \frac{h_{1}}{g(1)} \sum_{i=1}^{N-1} h_{i+1} \beta_{i+1} e^{a k} f_{i}(k)\left|e_{i}^{n}\right|+h_{1}\left|r^{n+1}\right| .
\end{aligned}
$$

Hence we obtain

$$
\left\|e^{n+1}\right\|_{1, \phi} \leq h_{1}\left|r^{n+1}\right|+\sum_{i=1}^{N-1} h_{i} \phi\left(x_{i}\right)\left|e_{i}^{n}\right| e^{a k} A_{i}^{1} A_{i}^{3} .
$$

with

$$
A_{i}^{1}=f_{i}(k) \frac{h_{i+1}}{h_{i}}, A_{i}^{3}=\frac{1}{\phi\left(x_{i}\right)}\left(\phi\left(x_{i+1}\right)+h_{1} \frac{\beta_{i+1}}{g(1)}\right) .
$$

Remark that

- For any $k>0$ and any $i \geq 1$,

$$
A_{i}^{1} \leq 1
$$

- There exists $k_{0}>0$ such that for any $k<k_{0}$

$$
A_{i}^{3} \leq e^{k \lambda_{0}+C_{2} a k^{2}}
$$

Estimate (38) is proved in the first part of this proof. To prove (39), we recall that

$$
g(x) \phi^{\prime}(x)+\beta(x)=\lambda_{0} \phi(x)
$$

so we get

$$
\begin{aligned}
A_{i}^{3} & =1+\frac{1}{\phi\left(x_{i}\right)}\left(\phi\left(x_{i+1}\right)-\phi\left(x_{i}\right)+\frac{h_{1}}{g(1)}\left(\lambda_{0} \phi\left(x_{i+1}\right)-g\left(x_{i+1}\right) \phi^{\prime}\left(x_{i+1}\right)\right)\right) \\
& =1+\frac{1}{\Phi\left(t_{i}\right)}\left(\Phi\left(t_{i+1}\right)-\Phi\left(t_{i}\right)+\frac{h_{1}}{g(1)}\left(\lambda_{0} \Phi\left(t_{i+1}\right)-\Phi^{\prime}\left(t_{i+1}\right)\right)\right)
\end{aligned}
$$

where $\Phi=\phi \circ x_{p}$. As $\frac{h_{1}}{g(1)}=k+a k^{2} C_{1}$ and as $\phi\left(t_{i}\right) \geq \frac{m b^{\alpha}}{\lambda_{0}}$, we obtain that for $k$ small enough

$$
A_{i}^{3} \leq e^{k \lambda_{0}+C_{2} a k^{2}} .
$$

The estimate (37) becomes for $k \leq k_{0}$ :

$e^{-\left(\lambda_{0}+C_{2} a k\right) t_{n+1}}\left\|e^{n+1}\right\|_{1, \phi} \leq 2 a k \ln (b) e^{-\left(\lambda_{0}+C_{2} a k\right) t_{n+1}} r_{n+1}+e^{-\left(\lambda_{0}+C_{2} a k\right) t_{n}}\left\|e^{n}\right\|_{1, \phi}$. 
Using a discrete Gronwall lemma we get:

$$
\begin{aligned}
\left\|e^{n}\right\|_{1, \phi} & \leq e^{\left(\lambda_{0}+C_{2} a k\right) t_{n}} 2 a \ln (b) \sum_{l=1}^{n} k e^{-\left(\lambda_{0}+C_{2} a k\right) t_{l}} r_{l} \\
& \leq 2 a \ln (b) k \frac{e^{\left(\lambda_{0}+C_{2} a k\right) t_{n}}-1}{e^{\left(\lambda_{0}+C_{2} a k\right) k}-1} \max _{l=1, \ldots, n} r_{l} \\
& \leq \frac{2 a \ln (b)}{\lambda_{0}} e^{\left(\lambda_{0}+C_{2} a k\right) t_{n}} \max _{l=1, \ldots, n} r_{l} .
\end{aligned}
$$

We conclude the proof using Proposition 23.

\subsection{Numerical results}

4.3.1. Influence of the production of metastases by metastases previously created

The figure 1 and figure 2 gives some comparisons between $N_{b_{\text {min }}}$ and $N_{\rho}$ (see (33) for their definition) for different times. We observe that the influence of the previously created metastases, that is to say the influence of the integral term in the boundary condition, is hardly visible before almost three years, as we could expect.
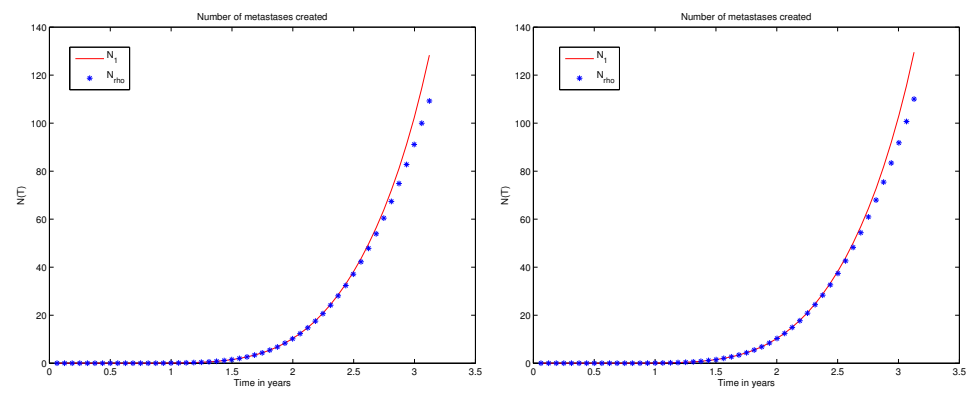

Figure 1. Number of metastases after 3 years with $k=2$ hours (left) and $k=1$ hours. (right)

\subsubsection{Evolution of the metastases number following the tumor size}

We compare the evolution of the metastases number for large size tumors. The figure 3 shows that before three years, there are very few metastases greater than $10^{8}$ cells. In the figure 4 , we compare the number of metastases $N_{10^{8}}$ and $N_{10^{9}}$. In these figures the time step is $k=1$ hour. 


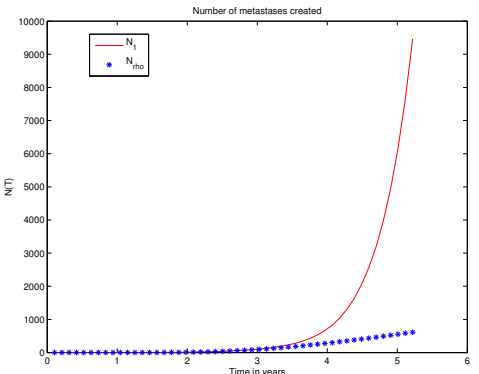

Figure 2. Number of metastases after 5 years

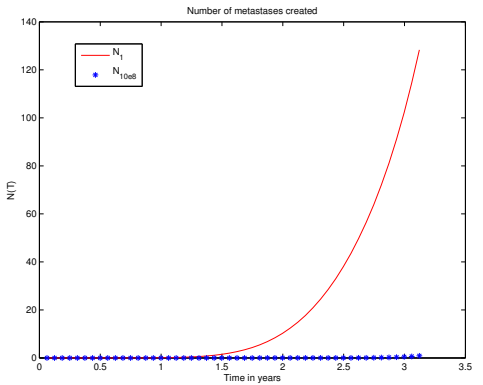

Figure 3. Comparison of the total number of metastases and the number of metastases larger than $10^{8}$ cells after 3 years

4.3.3. Asymptotic behaviour of $N_{1}$ and comparison with theoritical results

We compare in table 1 the values of $N_{1}(T)$, that we obtain with the one obtained in [11]. We observe a good agreement between our results.

\begin{tabular}{|c|c|c|}
\hline Time in years & $N_{1}(T)[11]$ & $N_{1}(T)$ authors \\
\hline$T=3$ & 135 & 134 \\
\hline$T=3.4$ & 263 & 260 \\
\hline$T=3.6$ & 396 & 396 \\
\hline$T=3.8$ & 712 & 718 \\
\hline
\end{tabular}

Table 1. Comparative results of $N_{1}(T)$

Let us now check the lower bound of $N_{1}$ given in (34). We choose two differents values of $a$ (see [11]) and we compute the approximation of $\lambda_{0}$ obtained in [11] and [6] by some Laplace transformations. In the figure 5, we observe that the function $\ln \left(N_{1}(t)\right)$ becomes linear after some time. These slope gives a very close approximation of $\lambda_{0}$, as shown in Table 2 : 

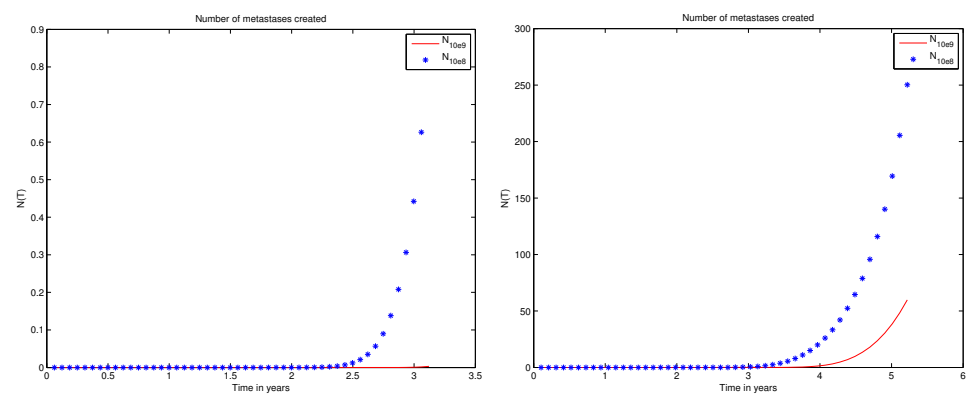

Figure 4. Comparison of the number of metastases larger than $10^{8}$ cells and larger than $10^{9}$ cells after 3 and 5 years

\begin{tabular}{|c|c|c|}
\hline $\mathrm{a}$ & $\lambda_{0}$ estimated in [1]] & $\lambda_{0}$ obtained with scheme (35) \\
\hline$a=.00286$ & 0.058 & 0.055 \\
\hline$a=.0143$ & 0.0201 & 0.0204 \\
\hline
\end{tabular}

Table 2. Comparative results of $\lambda_{0}$

In figure 6 , we compare the solution obtained by the characteristics scheme and the theoritical profile given in [6] and [11]:

$$
v(x, t) \simeq \frac{a}{m b^{\alpha} \ln b} \frac{1}{x} e^{\lambda_{0} t}\left(1-\frac{\ln x}{\ln b}\right)^{\frac{\lambda_{0}}{a}-1} \frac{1}{c\left(\lambda_{0}\right)}, x \in\left[1, x_{p}(t)\right]
$$

where

$$
c\left(\lambda_{0}\right)=\sum_{n=0}^{\infty} \frac{(-\alpha \ln b)^{n}}{n !\left(\frac{\lambda_{0}}{a}+n\right)^{2}} .
$$
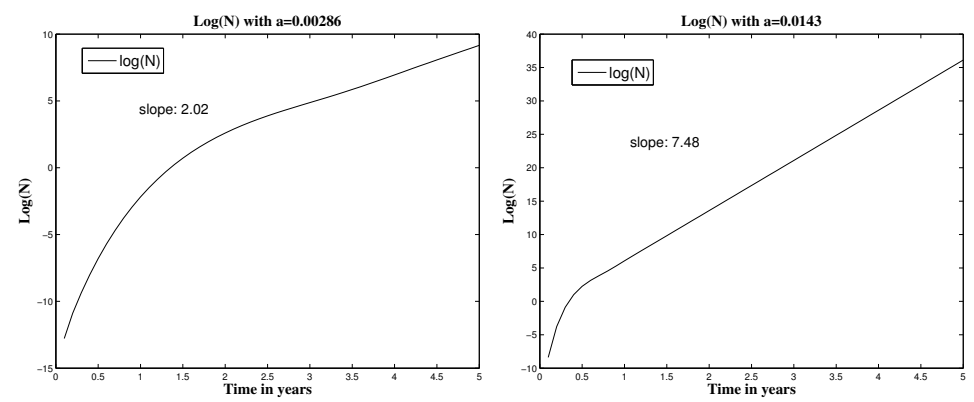

Figure 5. Log of the number of metastases after 5 years with $a=.00286$ (left) and $a=.0143$ (right). The asymptotic slope of these curves corresponds to $\lambda_{0} * 365$. 


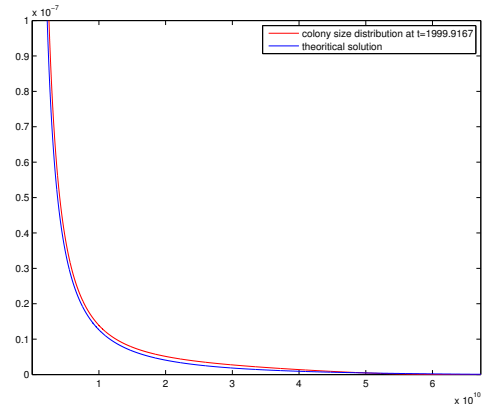

Figure 6. Comparison of the approximate solution and the theoritical solution after 2000 days with $\lambda_{0}=2.02995$

For $\alpha=.4$, we observed in figure 7 the "U-shaped" profile claimed in [11].

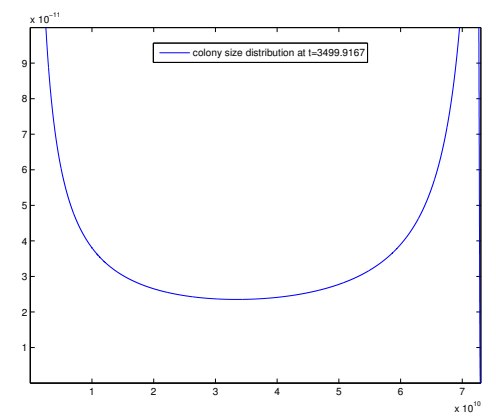

Figure 7. Solution with $\alpha=.4$

\section{Conclusion}

Even if this model is a simplification of a more complicated phenomena, its asymptotic gives interesting informations about the evolution of metastatic sites. In particular it shows that for large time, the exponential growth of the density of metastases is given by a parameter $\lambda_{0}$. The knowledge of this parameter is therefore fundamental. As its defintion shows (see (11)) , $\lambda_{0}$ depends on $a, b, \alpha, m$. An interesting problem would be to identify these coefficents by suitable observations on the patient. The first question could be: can we find a one to one correspondance between clinical observations and the parameters $a, b, \alpha, m$ that we use in the model? This leads to a mathematical uniqueness problem that we plan to investigate. The minimization of $\lambda_{0}$ 
with respect to the parameters $a, m, \alpha$ is another important question. One can define $H(\lambda, a, m, \alpha):=\int_{0}^{+\infty} \beta\left(G^{-1}(x)\right) e^{-\lambda_{0} x} d x$. Computing $G$ leads to

$$
H(\lambda, a, m, \alpha):=m b^{\alpha} \int_{0}^{+\infty} b^{-\alpha e^{-a x}} e^{-\lambda x} d x .
$$

Direct computations show that $H\left(\lambda_{0}, a, m, \alpha\right)=1$.

The Malthus parameter $\lambda_{0}$ is then an increasing function of all the parameters $a, m, \alpha$.

It would be interesting to obtain a sharper estimate where dependence on these parameters is expressed. Finding theoretical estimates is an open problem.

\section{Appendix}

We finally give in this section the proof of a technical result that we previously used:

Lemma 25. For any function $v \in L^{1}(1, b)$ such that $g v \in W^{1,1}(1, b)$, we have:

$$
\int_{1}^{b} \partial_{x}(g v) j(v) d x=-g(1)\|v\|_{L^{1}(1, b)}|v(1)| .
$$

Proof. Let us consider the following sequence:

$$
\Gamma_{n}(s)=\left\{\begin{array}{c}
1 \text { if } s \geq \frac{1}{n} \\
-1 \text { if } s \leq-\frac{1}{n} \\
n s \text { otherwise }
\end{array},\right.
$$

For any function $v \in D(A)$, we have $g \partial_{x} v \in L^{1}(1, b)$ and $g \partial_{x} v \Gamma_{n}^{\prime}(v) \in L^{1}(1, b)$. Furthermore $\left\{x \in(1, b) ; \Gamma_{n}^{\prime}(v(x)) \neq 0\right\}=\left\{|v| \leq \frac{1}{n}\right\}$. Hence $v g \partial_{x} v \Gamma_{n}^{\prime}(v) \in$ $L^{1}(1, b)$ and

$$
\int_{1}^{b} \partial_{x}(g v) \Gamma_{n}(v)=-\int_{1}^{b} v g \partial_{x} v \Gamma_{n}^{\prime}(v) d x-g(1) v(1) \Gamma_{n}(v(1)) .
$$

By Lebesgue's theorem, one can deduce:

$$
\partial_{x}(g v) \Gamma_{n}(v) \underset{n \rightarrow+\infty}{\longrightarrow} \partial_{x}(g v) \frac{j(v)}{\|v\|_{L^{1}(1, b)}}
$$

and moreover

$$
\Gamma_{n}(v(1)) \underset{n \rightarrow+\infty}{\longrightarrow}|v(1)| .
$$

Moreover, as $g \partial_{x} v \in L^{1}(1, b)$, we conclude that

$$
g\left(\partial_{x} v\right) v \Gamma_{n}^{\prime}(v) \underset{n \rightarrow+\infty}{\longrightarrow} 0 \text { in } L^{1}(1, b) .
$$




\section{References}

[1] O. Angulo and J. C. Lopez-Marcos. Numerical scheme for size-structured populations equations. Mathematical Biosciences, 157:169-188, 1999.

[2] H. T. Banks and F. Kappel. Transformation semigroups and $l^{1}$ approximation for size structured population models. Semigroup Forum, 38, 1989.

[3] D. Barbolosi and A. Iliadis. Optimizing drug regimens in cancer chemotherapy by an efficacy-toxicity model. Computer and Biomedical Research, 33, 2000.

[4] K.B. Bischoff, K.J. Himmelstein, R.L. Dedrick, and D.S. Zaharko. Phamarcokinetics and cell population growths models in cancer chemotherapy. Chem. Eng. Med. Biol, 118:47-64, 1973.

[5] J. Clairambault, B. Laroche, S. Mischler, and Perthame B. A mathematical model of the cell cycle and its control. Technical Report 4892, INRIA, France, July 2003.

[6] A. Devys, T. Goudon, and P. Lafitte. A model describing the growth and the size distribution of multiple metastatic tumors. Preprint, 2007.

[7] Post-Meeting Edition, editor. A mechanistic model predicting hematopoiesis and tumor growth to optimize docetaxel + epirubicin (ET) administration in metastatic breast cancer $(M B C)$ : Phase I trial, volume 25 (18S). ASCO Annual Meeting Proceedings, 2007, June 20 Supplement.

[8] K.J. Engen and R. Nagel. One parameter Semigroups for Linear Evolution Equations. Springer, 2000.

[9] Early Breast Cancer Trialists Collaborative Group. Systemic treatment of early breast cancer by hormonal, systemic or immune therapy: 133 randomized trials involving 31,000 recurrences and 24,000 deaths among 75,000 women. Lancet, 339:71-85, 1992.

[10] J. Harris, M. Morrow, and L. Norton. Malignant tumors of the breast. In Jr VT DeVita, S Hellman, and SA Rosenberg, editors, Cancer: Principles and Practice of Oncology (5th Edition), pages 1557-1602, Philadelphia: Lippincott-Raven, 1997.

[11] K. Iwata, K. Kawasaki, and N. Shigesad. A dynamical model for the growth and size distribution of multiple metastatic tumors. J. Theor. Biol, 203:177-186, 2000.

[12] P. Michel, S. Mischler, and Perthame B. General relative entropy inequality: an illustration on growth models. J. Math. Pures Appl, 84(9):1235$1260,2005$. 
[13] L. Norton. Theorical concepts and the emerging role of taxanes in adjuvant therapy. The Oncologist, 6(suppl3):30-35, 2001.

[14] A. Pazy. Semigroups of Linear Operators and Applications to Partial Differential Equations. Springer, 1983. Applied mathematical sciences.

[15] B. Perthame. Transport Equations on Biology. Birkhäuser, 2007. Frontiers in Mathematics. 